\title{
Evaluation of Rice Responses to the Blast Fungus Magnaporthe oryzae at Different Growth Stages
}

Xinglong Chen, Department of Plant Pathology, China Agricultural University, Beijing, 100193, China; Yulin Jia, ${ }^{\dagger}$ USDA-ARS, Dale Bumpers National Rice Research Center, Stuttgart, AR 72160, U.S.A; and Bo Ming Wu, ${ }^{\dagger}$ Department of Plant Pathology, China Agricultural University, Beijing, 100193, China

\begin{abstract}
Rice blast, caused by the fungus Magnaporthe oryzae, is the most damaging disease for rice worldwide. However, the reactions of rice to $M$. oryzae at different growth stages are largely unknown. In the present study, two temperate japonica rice cultivars, M-202 and Nipponbare, were inoculated synchronously at different vegetative growth stages, V1 to V10. Plants of M-202 at each stage from V1 to reproductive stage R8 were inoculated with $M$. oryzae race (isolate) IB-49 (ZN61) under controlled conditions. Disease reactions were recorded 7 days postinoculation by measuring the percentage of diseased area of all leaves, excluding the youngest leaf. The results showed that the plants were significantly susceptible at the V1 to V4 stages with a disease severity

of 26.7 to $46.8 \%$ and disease index of 18.62 to 37.76 for M-202. At the V1 to V2 stages, the plants were significantly susceptible with a disease a severity of 28.6 to $39.3 \%$ and disease index of 23.65 to 29.82 for Nipponbare. Similar results were observed when plants of M-202 were inoculated at each growth stage with a disease severity of 29.7 to $60.6 \%$ and disease index of 21.93 to 59.25 from V1 to V4. Susceptibility decreased after the V5 stage (severity $4.6 \%$ and index 2.17) and became completely resistant at the V9 to V10 stages and after the reproductive stages, suggesting that plants have enhanced disease resistance at later growth stages. These findings are useful for managing rice blast disease in commercial rice production worldwide.
\end{abstract}

Rice, a major food crop, is grown worldwide. Rice blast disease, caused by Magnaporthe oryzae, usually causes 5 to $30 \%$ yield loss (Skamnioti and Gurr 2009). M. oryzae is known to infect rice plants from the seedling to grain formation stages, causing leaf, nodal, collar, neck, or panicle blast (Webster and Gunnell 1992). Presently, blast disease is controlled by using genetic resistance, cultural practices, and/or fungicides. About 1 to $2 \%$ of the world rice production is grown in the United States, and the cost to control rice blast in the United States is estimated to be $\$ 61$ million annually (Nalley et al. 2016).

Plants have evolved sophisticated mechanisms to prevent damage caused by pathogens. One mechanism is to react to pathogens differently at different growth stages. It is well known that younger plants are more susceptible to diseases than older plants (Century et al. 1999; Goto et al. 1961; Hunter et al. 1978; Kahn and Libby 1958; Koch and Mew 1991; Lazarovits et al. 1981; Parniske et al. 1997; Pretorius et al. 1988; Roumen et al. 1992; Wang et al. 2006). Increased resistance owing to aging has been referred to as one mechanism of partial resistance (Kahn and Libby 1958). Disease resistance observed in adult plants has been referred to as adult plant resistance (APR), and its impact on rice yield was noted in the 1980s (Hwang et al. 1987; Kim et al. 1987).

The vegetative (V1 to V13) and reproductive (R0 to R9) growth stages of rice were previously described by Counce et al. (2000). Blast disease surveillance has relied on scouting rice fields after rice is permanently established, either after transplanting or direct seeding worldwide. However, scouting occasionally fails to predict blast epidemics, which often result in significant crop losses. A detailed study of rice blast development under defined growth

${ }^{\dagger}$ Corresponding authors: Yulin Jia; E-mail: yulin.jia@ars.usda.gov and Bo Ming Wu; E-mail: bmwu@cau.edu.cn

Funding: Funding was provided by the China Scholarship Council (CSC).

*The $\boldsymbol{e}$-Xtra logo stands for "electronic extra" and indicates that two supplementary tables are published online.

Accepted for publication 11 July 2018.

This article is in the public domain and not copyrightable. It may be freely reprinted with customary crediting of the source. The American Phytopathological Society, 2019. stages is urgently needed to guide the time of scouting. To date, the specific growth stages for inoculations with $M$. oryzae are often not reported (Ashikawa et al. 2008; Bryan et al. 2000). For example, Challagulla et al. (2015) inoculated 8-, 12-, and 16week-old rice plants to evaluate rice blast resistance. Conversely, Sallaud et al. (2003) inoculated seedlings 3 weeks after sowing for disease evaluation and quantitative trait locus mapping. The number of days after sowing does not necessarily reflect a specific growth stage of the same genotype under different environments or different seasons. Under controlled conditions, it is speculated that rice seedlings of the same genotype at different growth stages would react to $M$. oryzae differently. If so, different reactions would be expected after inoculations at different growth stages. To date, enhanced resistance of adult rice plants to $M$. oryzae at different growth stages as described by Counce et al. (2000) under controlled environmental conditions has not been documented.

The objectives of the present study were to determine (i) the reactions of two rice cultivars, M-202 and Nipponbare, when inoculated at growth stages V1 to V10 with $M$. oryzae at the same time and (ii) the reactions of one cultivar, M-202, inoculated individually at different growth stages from V1 to R8.

\section{Materials and Methods}

Rice materials and growth. The temperate (California) japonica rice cultivar M-202 (PI 494105) and the genome sequence reference temperate japonica cultivar Nipponbare (both have a growth duration of about 110 days from planting to harvesting) were used in the experiments, and both are susceptible to the U.S. M. oryzae race IB-49. Seeds were germinated on moistened Whatman filter papers (GE Healthcare, Pittsburgh, PA, U.S.A.) in Petri dishes for 3 days at $30^{\circ} \mathrm{C}$ in a dark incubator. The germinated seeds were transferred to black plastic pots $(12.8 \mathrm{~cm}$ diameter at top, $12.0 \mathrm{~cm}$ depth, and $9.5 \mathrm{~cm}$ at the bottom with eight holes). A total of eight pots with local silt loam soil were randomly placed in a tray $(25.4 \times 50.8 \mathrm{~cm})$ and transferred to a greenhouse at 24 to $30^{\circ} \mathrm{C}$ with a 16-h light and 8-h dark daily light cycle. Germinated seeds were sowed every 3 to 4 days for the early vegetative growth stages and every 7 to 8 days for the late vegetative growth stages and reproductive growth stages (Counce et al. 2000). One to two grams of controlled-release fertilizer Osmocote Plus (15-9-12 [N-P-K], Scotts, Marysville, OH, U.S.A.) was added to each pot after the V2 stage. Weaker seedlings were removed to maintain seedling 
uniformity in each pot for synchronous and individual stage inoculation. Rice plants at different growth stages were tagged as soon as the prophyll leaves emerged (Figs. 1 and 2). Rice seeds were sown at different times to achieve specific stages during inoculation. The plants at different growth stages that were inoculated at the same time are referred to hereafter as synchronous inoculation.

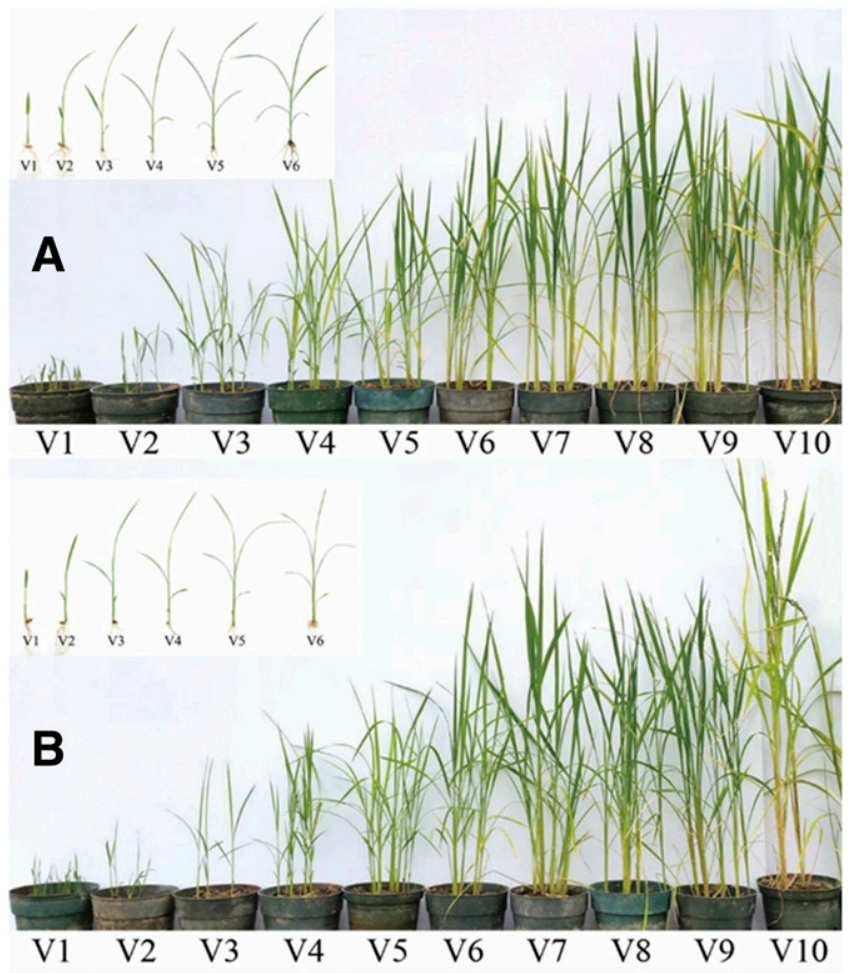

Fig. 1. Rice plants with growth stages indicated before inoculation with IB-49 (ZN61). A, M-202 (V1 to V10); seedlings in the upper left corner indicate single M-202 plants from stages V1 to V6. B, Susceptible cultivar Nipponbare (V1 to V10); seedlings in the upper left corner indicate single plants of Nipponbare from stages $\mathrm{V} 1$ to $\mathrm{V} 6$.
M. oryzae race and culture. Race IB-49 (isolate ZN61; Correll et al. 2000) of M. oryzae was used for the inoculation of M-202 and Nipponbare. Stored desiccated filter paper pieces containing fungal mycelium and spores were removed from a $-20^{\circ} \mathrm{C}$ freezer, placed on oatmeal agar plates, and incubated under black-white fluorescent light for 3 to 5 days at $23^{\circ} \mathrm{C}$. The plugs were then transferred to new oatmeal agar plates for another 7 to 10 days at $24^{\circ} \mathrm{C}$ with $45 \%$ relative humidity to produce conidia. Conidia were harvested with sterilized $0.25 \%$ gelatin (Thermo Fisher Scientific, Asheville, NC, U.S.A.) solution using sterile cotton-tipped applicators and filtered through four layers of cheesecloth. The concentration of conidia was determined using a hemocytometer under a microscope and adjusted with sterilized $0.25 \%$ gelatin to $1 \times 10^{5}$ conidia/ml.

Inoculation and evaluation. Rice plants of both cultivars were inoculated with $M$. oryzae by spraying a spore suspension at a concentration of $1 \times 10^{5}$ conidia/ml in a $0.25 \%$ gelatin solution according to the procedure described by Valent et al. (1986) with slight modifications. The seedlings were sprayed from the top and sides to ensure that all the leaf surfaces were covered with a similar amount of inoculum. All healthy green leaves were sprayed with the inoculum at the reproductive stages. Specifically, for the synchronous experiment, three to eight plants of the two cultivars at each of the growth stages from V1 to V10 (Counce et al. 2000) were selected and placed in trays filled with 1 to 2 liters of water. Trays containing seedlings at the V1 to V5 stages were moved into a black plastic bag and sprayed with $15 \mathrm{ml}$ of $1 \times 10^{5}$ conidia/ml. Trays with larger plants at the V6 to V10 stages were placed in a separate plastic bag, sprayed with $30 \mathrm{ml}$ of inoculum, and for negative controls, only $0.25 \%$ gelatin in a separate bag. In each case, conidia and gelatin controls were applied with an airbrush sprayer (Paasche IVL double action airbrush; Paasche Airbrush, Chicago, IL, U.S.A.) connected to compressed air at $30 \mathrm{psi}$. After each inoculation, the bags were sealed for $24 \mathrm{~h}$ to maintain humidity for the conidia to germinate and penetrate the cuticle of the plant (Howard and Ferrari 1989). The experiment was carried out four times.

For another experiment, cultivar M-202 was inoculated with 5 to $10 \mathrm{ml}$ of $1 \times 10^{5}$ conidia/ml (depending on size of plant) at each growth stage from V1 to R8. After each inoculation, the plants were removed from the plastic bags and placed in a growth chamber with

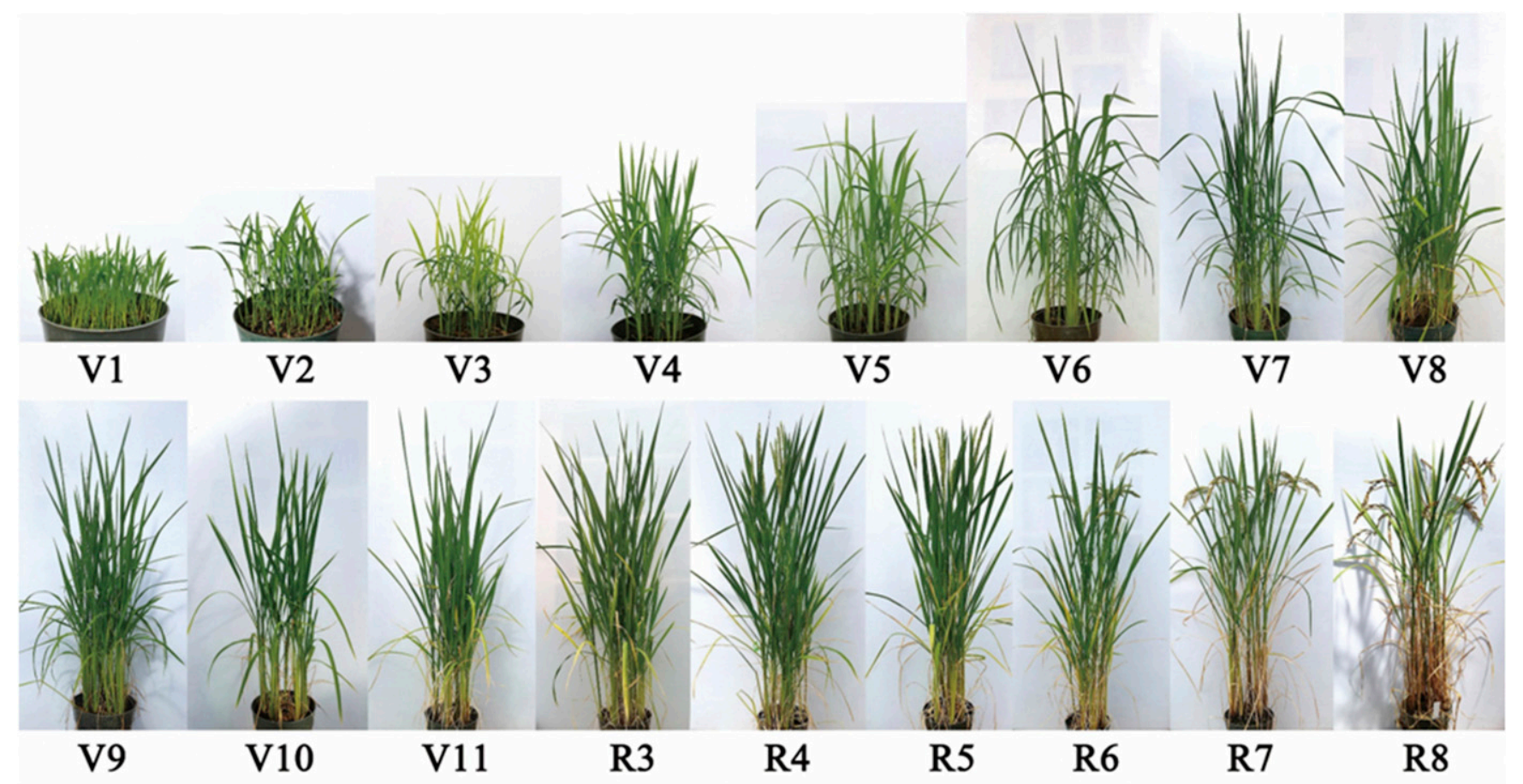

Fig. 2. Rice cultivar M-202 with growth stages from V1 to R8 before individual inoculations with IB-49 (ZN61). 
automatic humidity and temperature controls. The relative humidity was $80 \%$ at $24^{\circ} \mathrm{C}$ with a 16 -h light and 8 -h dark daily light cycle. Plants remained in the growth chamber until typical disease symptoms were observed approximately 6 to 7 days after inoculation. Symptoms were evaluated 2 weeks after inoculation. Disease reactions were evaluated with the following methods: for plants that were inoculated synchronously (growth stages V1 to V10), a visual estimation of the percentage of the areas of the leaves with lesions, ranging from 0 to $100 \%$, was used to evaluate the second youngest leaf of each plant. For plants that were inoculated at each growth stage, all inoculated leaves except the youngest were scored with the same rating scale as the synchronous inoculation. Disease incidence (DI) was recorded as the percentage of infected leaves out of all leaves except the youngest. Disease severity (DS) was calculated as the percentage of disease lesion area divided by the total area of infected leaves. Average severities were then calculated for all infected leaves in each treatment, and disease index was calculated as the product of DI and DS.

$$
\begin{gathered}
\text { DI }(\%)=\frac{\text { number of diseased leaves }}{\text { total number of leaves assessed }} \times 100 \\
\text { DS }(\%)=\text { average }\left(\frac{\text { lesion area }}{\text { total area of infected leaves }}\right) \times 100 \\
\text { disease index }=\text { DI } \times \text { DS } \times 100
\end{gathered}
$$

Experimental design. All experiments were performed under controlled environmental conditions. Because the leaf area of rice plants differs greatly from V1 to R8, for the synchronous inoculation, three to eight seedlings at each growth stage were maintained in each pot based on the size of the plants. Fewer seedlings were used for larger plants, and more seedlings were used for smaller plants. The experiment was carried out four times with two susceptible cultivars: M-202 and Nipponbare. For the experiment in which the specific stages of cultivar M-202 were inoculated, the number of plants for each stage varied as follows: V1, 183; V2, 77; V3, 27; V4, 25; V5, 23; V6, 19; V7, 18; V8, 16; V9, 14; V10, 8; R1, 10; R2, 9; R3, 10; R4, 9; R5, 7; R7, 6; and R8, 6.

Statistical analysis. Because DI and DS did not follow normal distributions and their variances depended on the means, the GLIMMIX procedure, which does not require stringent normal distribution and homogeneity of variance, was used to perform analysis of variance in SAS (version 9.4, SAS Institute, Cary, NC, U.S.A.). The effects of different rice growth stages and cultivars on DI, DS, and index were tested. For the effects that were statistically significant, mean comparisons were done using the LSMEANS statement in the GLIMMIX procedure.

\section{Results}

Reactions of plants simultaneously inoculated at growth stages from V1 to V10. Analysis of variance revealed that DI, DS, and

Table 1. Type III test of fixed effects of rice cultivars (M-202 and Nipponbare) and rice growth stages (V1 to V10) on disease incidence (DI), severity (DS), and index at inoculation

\begin{tabular}{llcccc}
\hline Parameter & \multicolumn{1}{c}{ Effect } & Num DF $^{\mathbf{a}}$ & Den DF & $\boldsymbol{F}$ value & Pr $>\boldsymbol{F}^{\mathbf{b}}$ \\
\hline DI & Stage & 9 & 60 & 6.25 & $<0.0001$ \\
& Cultivar & 1 & 60 & 0.00 & 0.999 \\
\multirow{4}{*}{ DS } & Stage $\times$ cultivar & 9 & 60 & 0.73 & 0.6812 \\
& Stage & 9 & 60 & 37.75 & $<0.0001$ \\
& Cultivar & 1 & 60 & 20.10 & $<0.0001$ \\
Index & Stage $\times$ cultivar & 9 & 60 & 2.68 & 0.011 \\
& Stage & 9 & 60 & 15.03 & $<0.0001$ \\
& Cultivar & 1 & 60 & 3.29 & 0.0049 \\
& Stage $\times$ cultivar & 9 & 60 & 0.8 & 0.1496 \\
\hline
\end{tabular}

a Num DF = degrees of freedom numerator; and Den DF = degrees of freedom denominator.

${ }^{\mathrm{b}}$ A value smaller than 0.01 denotes a significant effect of the factor (the original data are listed in Supplementary Table S1). index were significantly affected by growth stages from V1 to V10 during inoculation, and both the DI and DS decreased from early growth stages to later stages for both rice cultivars (Tables 1 and 2 ). However, there was no significant effect of the stage $\times$ cultivar interaction in the DI and disease index, suggesting that the cultivar did not change the decreasing trend of the DI and disease index as the growth stage increased. However, the cultivar had some insignificant effect on the trend of DS $(P=0.011$, Table 1$)$.

For cultivar M-202, the leaves at all growth stages except V10 were susceptible to IB-49 (ZN61) and showed typical leaf blast symptoms (Fig. 3). There was a trend that suggested that the later the growth stage, the less they were susceptible (Fig. 4A and B). The leaves at the V1 to V4 stages were highly susceptible with a DI of 69.1 to $80.8 \%$, DS of 26.7 to $46.8 \%$, and disease index of 18.62 to 37.76 . The V5 stage had a high incidence $(63.3 \%)$ but a significantly lower severity $(7.4 \%)$ than the V1 to V4 stages $(\geq 26.7 \%)$. Consequently, the disease index for the V5 stage (3.97) was also significantly lower than the V1 to V4 ( $\geq 18.62)$ stages. The number and

\begin{tabular}{|c|c|c|c|c|c|c|}
\hline $\begin{array}{l}\text { Growth } \\
\text { stage }\end{array}$ & $\begin{array}{l}\text { Inoculum } \\
\text { volume } \\
(\mathrm{ml})\end{array}$ & $\begin{array}{l}\text { Assessed } \\
\text { leaves }^{\mathbf{a}}\end{array}$ & $\begin{array}{l}\text { Diseased } \\
\text { leaves }^{\mathbf{a}}\end{array}$ & $\begin{array}{c}\text { DI } \\
(\times \mathbf{1 0 0})^{\mathbf{b}}\end{array}$ & $\begin{array}{c}\text { DS } \\
(\times 100)^{\mathbf{c}}\end{array}$ & $\begin{array}{l}\text { Disease } \\
\text { index }^{d}\end{array}$ \\
\hline V1 & 10 & 183 & 179 & 97.8 & 60.6 & 59.25 \\
\hline V2 & 5 & 153 & 144 & 94.1 & 48.3 & 45.44 \\
\hline V3 & 10 & 80 & 55 & 68.8 & 32.5 & 22.34 \\
\hline V4 & 5 & 99 & 73 & 73.7 & 29.7 & 21.93 \\
\hline V5 & 5 & 91 & 43 & 47.3 & 4.6 & 2.17 \\
\hline V6 & 10 & 94 & 26 & 27.7 & 2.5 & 0.69 \\
\hline V7 & 10 & 89 & 10 & 11.2 & 2.0 & 0.22 \\
\hline V8 & 10 & 95 & 34 & 35.8 & 2.3 & 0.82 \\
\hline V9 & 10 & 94 & 0 & 0.0 & 0.0 & 0.00 \\
\hline V10 & 10 & 57 & 0 & 0.0 & 0.0 & 0.00 \\
\hline V11 & 20 & 75 & 7 & 9.3 & 1.3 & 0.12 \\
\hline R3 & 20 & 66 & 0 & 0.0 & 0.0 & 0.00 \\
\hline R4 & 20 & 72 & 0 & 0.0 & 0.0 & 0.00 \\
\hline R5 & 20 & 65 & 0 & 0.0 & 0.0 & 0.00 \\
\hline R6 & 20 & 45 & 0 & 0.0 & 0.0 & 0.00 \\
\hline R7 & 20 & 40 & 0 & 0.0 & 0.0 & 0.00 \\
\hline R8 & 20 & 38 & 0 & 0.0 & 0.0 & 0.00 \\
\hline
\end{tabular}

Table 2. Disease incidence (DI), severity (DS), and index of rice cultivar M-202 (V1 to R8) to Magnaporthe oryzae race IB-49 (isolate ZN61)

a Assessed leaves represent the number of leaves evaluated. All inoculated leaves except the youngest ones were evaluated. Diseased leaves and assessed leaves were evaluated with all inoculated leaves except the youngest one in individual inoculation from V1 to R8 using visual estimation of the percentage of disease lesion area from 0 to $100 \%$. (The original data are listed in Supplementary Table S2.)

${ }^{b} \mathrm{DI}$ is the percent of diseased leaves.

${ }^{c}$ DS is the percent area of lesions, estimated from 0 to $100 \%$. See Supplementary Table $\mathrm{S} 2$ for original data.

${ }^{\mathrm{d}}$ Disease index $=\mathrm{DI} \times \mathrm{DS} \times 100$.
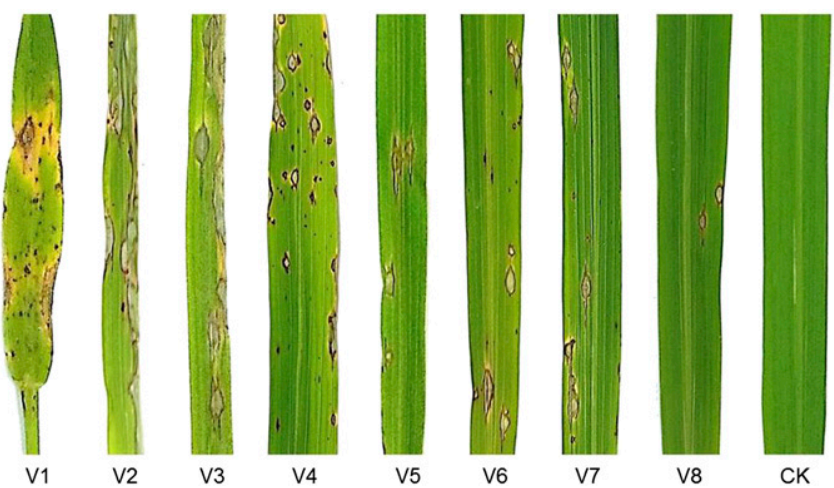

Fig. 3. Typical blast symptoms on rice leaves at indicated growth stages 1 week after inoculation. $\mathrm{CK}=$ check. 
size of lesions were reduced after the V6 stage with a DS ranging from 4.0 to $0.3 \%$ and disease index from 1.99 to 0.13 . After the V10 stage, lesions were not observed (Fig. 3).

Lesions on Nipponbare were observed throughout all growth stages except V10 (Fig. 4C and D). A decreasing trend with growth stage was also found for disease susceptibility. Growth stages V1 and V2 were the most susceptible stages to IB-49 with a significantly higher DS of 39.3 and $28.6 \%$ and a significantly higher disease index of 29.82 and 23.65, respectively. Growth stages V3 to V6 also had a relatively high DI ranging from 51.7 to $79.2 \%$, even though DS declined from 11.8 to $2.0 \%$ and disease index declined from 9.42 to 0.98 with clear lesions on the leaves. There were few symptoms after V7, and no lesions were observed at V10 (Fig. 3).

Disease reactions of plants inoculated at different growth stages from V1 to R8. The trend of disease reactions from V1 to V10 was highly similar to the trend found on the simultaneously inoculated plants (Fig. 5, Table 2). Plants became more resistant to $M$. oryzae as the plants got older. DI, DS, and disease index were high from V1 to V4 with 68.8 to $97.8 \%, 29.7$ to $60.6 \%$, and 21.93 to 59.25 , respectively. Among them, the V1 and V2 stages had a high DI of 97.8 and $94.1 \%$, DS of 60.6 and $48.3 \%$, and disease index of 59.25 and 45.44, respectively. After the V4 stage, DS and disease index reduced rapidly from 4.6 to $0 \%$ and 2.17 to 0.00 , respectively. DI fluctuated with a small peak at the V8 stage with a DI of $35.8 \%$ and a low disease index of 0.82 owing to the low DS of $2.3 \%$. Additionally, there was an extremely low DS of $1.3 \%$ and disease index of 0.12 at the V11 stage despite not observing disease reactions at V9 and V10.

\section{Discussion}

In the present study, we simultaneously inoculated rice plants from the V1 to V10 growth stages. A second group of plants were inoculated at different growth stages from V1 to R8. Leaf areas from V1 to V2 were small and about 140 times less than those measured at V10 (data not shown). For this reason, we inoculated the V1 to V5 stage seedlings with $15 \mathrm{ml}$ of $1 \times 10^{5}$ conidia/ml, whereas plants at V6 to $\mathrm{V} 10$ were sprayed with $30 \mathrm{ml}$ of the same concentration. Plants at V1 to V5 received more conidia per unit area than plants at V6 to V10. M-202 rice plants at growth stages ranging from V1 to R8 were inoculated with different suspension volumes to achieve similar inoculum per unit area (Table 2). DI and DS collected from plants were calculated from the average of multiple leaves of plants inoculated at the same time. M-202 rice plants that were inoculated at specific stages received more inoculum than for the synchronous inoculation (Table 2). Consequently, higher DS and disease index were observed for plants inoculated at each growth stage. Despite these inconsistencies, the conclusions drawn from both experiments were similar.

Cultivars M-202 and Nipponbare were completely resistant to $M$. oryzae at V10 with synchronous inoculation. This result was corroborated by inoculating cultivar M-202 at different growth stages separately from V1 to R8. The M-202 plants were resistant after V9 except for a few small lesions observed at V11. Plants at growth stages V1 to V8 were blast susceptible and became resistant at V9 and V10. Rice seedlings from V1 to V4 were extremely susceptible with a very high disease index, but their susceptibility declined after V5. Symptoms were not observed at V10 for plants inoculated at the same time, and symptoms were not observed at V9 for plants inoculated at each growth stage.

Resistance to $M$. oryzae increased in older leaves of rice (Goto et al. 1961; Kahn and Libby 1958; Roumen et al. 1992), and this is

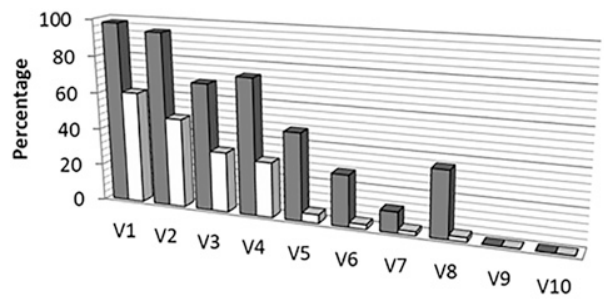

口Incidence (\%) 口Severity (\%)

Vegetative Growth Stages

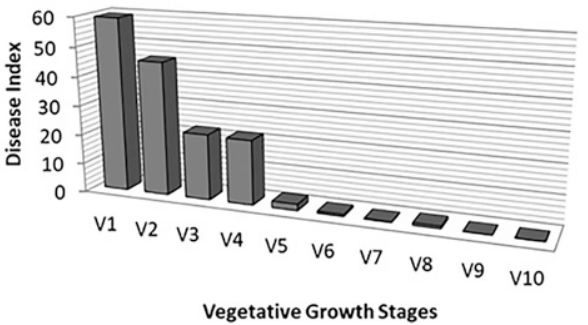

$\square$ Disease Index

Fig. 5. Histograms of rice blast disease incidence, severity, and index.
A

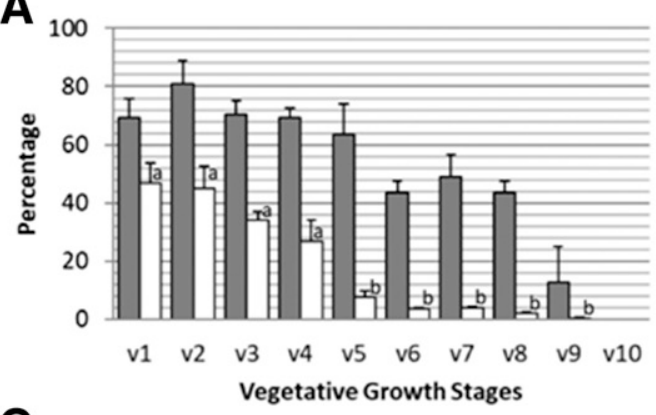

C

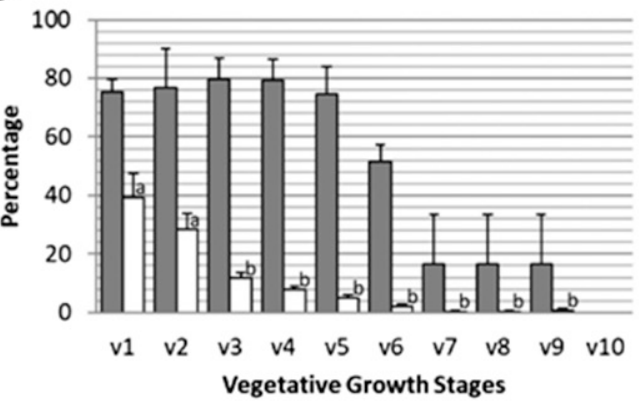

口Incidence (\%) 口Severity (\%)

口incidence (\%) 口Severity (\%)

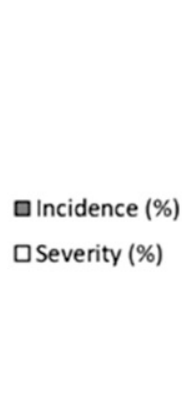

B

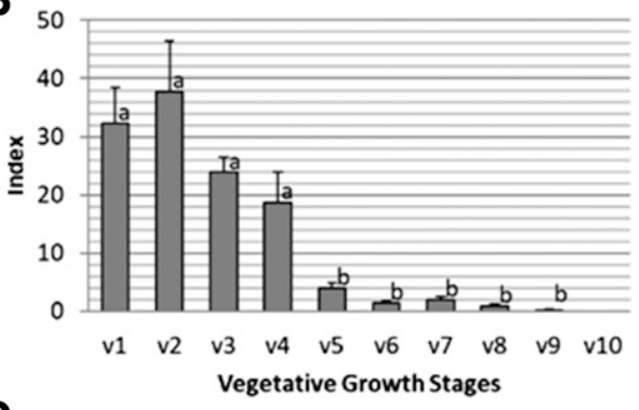

D

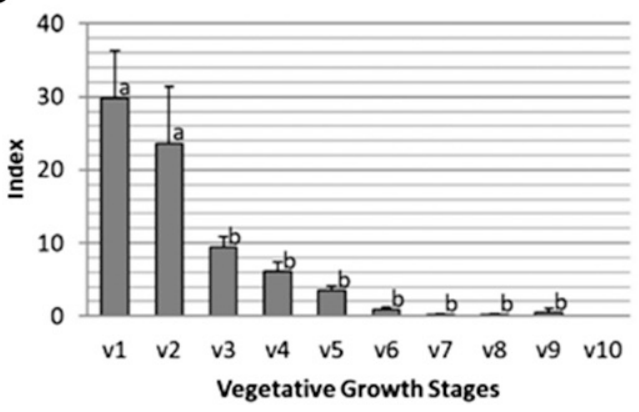

Disease Index

Disease Index

Fig. 4. Histograms of rice blast disease incidence, severity, and index. A and B, M-202 from V1 to V10 inoculated with IB-49 (ZN61). C and D, Nipponbare inoculated with IB-49 (ZN61). Data shown are the result of four independent experiments. 
consistent with the observation of increased partial resistance to leaf blast with increased leaf age (Kahn and Libby 1958). The major blast resistance $(R)$ genes are often constitutively expressed from seedling to adult growth stages, whereas APR genes function mainly at the adult stage (Hwang et al. 1987; Kim et al. 1987). To avoid confounding the effects of major $R$ genes, blast-susceptible rice cultivars were used for this study. The M-202 cultivar was more susceptible to blast infection than Nipponbare, especially at the V3 and V4 stages.

Previously, differential responses were observed among different cultivars, and the number of sporulating lesions $/ \mathrm{cm}^{2}$ of leaves declined with increasing leaf age in both genotypes (Roumen et al. 1992). The fraction of sporulating lesions $/ \mathrm{cm}^{2}$ of 1 -week-old leaves over 1- to 2-day-old leaves was about $25 \%$ in the susceptible cultivar CO39. The fraction of sporulating leaf area was $2 \%$ or less in the more resistant cultivars IR36, IR60, and IR62. The number of lesions in CO39 was about seven times that of IR60 (Roumen 1992; Roumen et al. 1992). In this study, we showed that plants were more resistant to blast at later growth stages. These findings suggest that older plants are more resistant to $M$. oryzae.

The rice blast life cycle starts from a single asexual spore and ends with a lesion containing thousands of spores within a week (Jia et al. 2016). In Arkansas, rice farmers often incur significant crop losses owing to panicle blast without evidence of leaf blast. This could be because scouting for blast in Arkansas often starts at the late tillering phase that is around the V9 growth stage. As demonstrated in this study, blast-susceptible rice plants often expressed APR after the V5 stage, which could mask the susceptibility of a rice cultivar. A single spore can infect any panicle of a susceptible rice cultivar, resulting in $100 \%$ grain loss of one panicle. Therefore, we suggest scouting for blast in commercial fields as soon as seedlings emerge: the best stages would be from V3 to V4. When rice seedlings are older than V5, APR appears to mask susceptibility, which may misguide fungicide application. Blast observed at the V11 growth stage, which is around the booting stage, suggests that APR was not strong enough to prevent $M$. oryzae infection. This is consistent with the fact that fungicide application is recommended at this stage to control panicle blast.

These data suggest that older plants are more resistant to $M$. ory$z a e$, and this finding is consistent with previous studies by others that demonstrated that older leaves of APR plants were more resistant to diseases, such as wheat to Puccinia triticina (Pretorius et al. 1988), soybean to Phytophthora megasperma var. sojae (Lazarovits et al. 1981), cotton to Rhizoctonia solani (Hunter et al. 1978), and tomato to Cladosporium fulvum (Parniske et al. 1997). The resistance of rice seedlings to bacterial leaf blight disease caused by Xanthomonas oryzae pv. oryzae progressively increased from the two-leaf stage to later stages with complete resistance on adult plants (Century et al. 1999; Koch and Mew 1991). These findings suggest that adult plants of certain cultivars have evolved more effective resistance responses to pathogens.

In summary, this study demonstrated that the two blast-susceptible rice cultivars react to $M$. oryzae similarly with increasing resistance at later growth stages. Plants from the V1 to V4 stages were susceptible. After the V5 growth stage, their susceptibility was reduced, and visible symptoms were found when inoculation was done at the V9 to V10 growth stages under highly homogenous controlled environmental conditions. This study demonstrated that blast-susceptible rice cultivars exhibit enhanced disease resistance at late growth stages and that it is of utmost importance to scout rice fields before the V5 growth stage to avoid building up blast spores before heading. Hopefully, these results will be useful for guiding research on the genetic basis of APR and for managing rice blast in commercial fields worldwide.

\section{Acknowledgments}

We thank the China Scholarship Council (CSC) for supporting Xinglong Chen's living and traveling expenses to the United States; X. Wang and H. Zhao for their advice on seedling growth and inoculation; James C. Correll, Department of Plant Pathology, University of Arkansas, Fayetteville, 72701, U.S.A., for inviting Xinglong Chen to the United States; and Mary S. Jia of the Henry K. Dupree Aquaculture Center and Tracy Bianco of the Dale Bumpers National Rice
Research Center for manuscript proofreading and fungal culture assistance. The United States Department of Agriculture (USDA) is an equal opportunity provider and employer

\section{Literature Cited}

Ashikawa, I., Hayashi, N., Yamane, H., Kanamori, H., Wu, J., Matsumoto, T. Ono, K., and Yano, M. 2008. Two adjacent nucleotide-binding site-leucinerich repeat class genes are required to confer $P i k m$-specific rice blast resistance. Genetics 180:2267-2276.

Bryan, G. T., Wu, K., Farrall, L., Jia, Y., Hershey, H. P., McAdams, S. A., Faulk, K. N., Donaldson, G. K., Tarchini, R., and Valent, B. 2000. A single amino acid difference distinguishes resistant and susceptible alleles of the rice blast resistance gene Pi-ta. Plant Cell 12:2033-2045.

Century, K. S., Lagman, R. A., Adkisson, M., Morlan, J., Tobias, R., Schwartz, K., Smith, A., Love, J., Ronald, P. C., and Whalen, M. C. 1999. Developmental control of Xa21-mediated disease resistance in rice. Plant J. 20:231-236.

Challagulla, V., Bhattatal, S., and Midmore, D. J. 2015. In-vitro vs in-vivo inoculation: Screening for resistance of Australian rice genotypes against blast fungus. Rice Sci. 22:132-137.

Correll, J. C., Harp, T. L., Guerber, J. C., Zeigler, R. S., Liu, B., Cartwright, R. D., and Lee, F. N. 2000. Characterization of Pyricularia grisea in the United States using independent genetic and molecular markers. Phytopathology 90 : 1396-1404.

Counce, P. A., Keisling, T. C., and Mitchell, A. J. A. 2000. A uniform, objective, and adaptive system for expressing rice development. Crop Sci. 40:436-443.

Goto, K., Hirano, K., and Ohata, K. 1961. Susceptibility of leaf of rice to blast in reference to leaf age and position. I. Variation of susceptibility among different leaf positions and grades of emergence of top leaf. Spec. Bull. Okayama Prefer. Agric. Exp. Stn. 58:77-88.

Howard, R. J., and Ferrari, M. A. 1989. The role of melanin in appressorium function. Exp. Mycol. 13:403-418.

Hunter, R. E., Halloin, J. M., Veech, J. A., and Carter, W. W. 1978. Terpenoid accumulation in hypocotyls of cotton seedlings during aging and after infection by Rhizoctonia solani. Phytopathology 68:347-350.

Hwang, B. K., Koh, Y. J., and Chung, H. S. 1987. Effects of adult-plant resistance on blast severity and yield of rice. Plant Dis. 71:1035-1038.

Jia, Y., Zhou, E., Lee, S., and Bianco, T. 2016. Co-evolutionary dynamics of rice blast resistance gene Pi-ta and Magnaporthe oryzae avirulence gene AVRPital. Phytopathology 106:676-683.

Kahn, R. P., and Libby, J. L. 1958. The effect of environmental factors and plant age on the infection of rice by the blast fungus, Piricularia oryzae. Phytopathology 48:25-30.

Kim, K. D., Hwang, B. K., and Kon, Y. J. S. 1987. Evaluation of rice cultivars under greenhouse conditions for adult-plant resistance to Pyicularia oryzae. J. Phytopathol. 120:310-316.

Koch, M. F., and Mew, T. W. 1991. Effects of plant age and leaf maturity on the quantitative resistance of rice cultivars to Xanthomonas campestris pv. oryzae. Plant Dis. 75:901-904.

Lazarovits, G., Stossel, P., and Ward, E. W. B. 1981. Age-related changes in specificity and glyceollin production in the hypocotyl reaction of soybeans to Phytophthora megasperma var. sojae. Phytopathology 71:94-97.

Nalley, L., Tsiboe, F., Durand-Morat, A., Shew, A., and Thoma, G. 2016 Economic and environmental impact of rice blast pathogen (Magnaporthe oryzae) alleviation in the United States. PLoS One 11:e0167295

Parniske, M., Hammond-Kosack, K. E., Golstein, C., Thomas, C. M., Jones, D. A., Harrison, K., Wulff, B. B. H., and Jones, J. D. G. 1997. Novel disease resistance specificities result from sequence exchange between tandemly repeated genes at the $C f-4 / 9$ locus of tomato. Cell 91:821-832.

Pretorius, Z. A., Rijkenberg, F. H. J., and Wilcoxson, R. D. 1988. Effect of growth stage, leaf position, and temperature on adult-plant resistance of wheat infected by Puccinia recondita f.sp. tritici. Plant Pathol. 37:36-44.

Roumen, E. C. 1992. Effect of leaf age on components of partial resistance in rice to leaf blast. Euphytica 63:271-279.

Roumen, E. C., Bonman, J. M., and Parlevliet, J. E. 1992. Leaf age related partial resistance to Pyricularia oryzae in tropical lowland rice cultivars as measured by the number of sporulating lesions. Phytopathology 82:1414-1417.

Sallaud, C., Lorieux, M., Roumen, E., Tharreau, D., Berruyer, R., Svestasrani, P., Garsmeur, O., Ghesquiere, A., and Notteghem, J.-L. 2003. Identification of five new blast resistance genes in the highly blast-resistant rice variety IR64 using a QTL mapping strategy. Theor. Appl. Genet. 106:794-803.

Skamnioti, P., and Gurr, S. J. 2009. Against the grain: Safeguarding rice from rice blast disease. Trends Biotechnol. 27:141-150.

Valent, B., Crawford, M. S., Weaver, C. G., and Chumley, F. G. 1986. Genetic studies of pathogenicity and fertility of Magnaporthe grisea. Iowa State J. Res. 60:569-594

Wang, Y. S., Pi, L. Y., Chen, X., Chakrabarty, P. K., Jiang, J., De Leon, A. L., Liu, G. Z., Li, L., Benny, U., Oard, J., Ronald, P. C., and Song, W. Y. 2006. Rice $\mathrm{XA} 21$ binding protein 3 is a ubiquitin ligase required for full $\mathrm{Xa} 21$-mediated disease resistance. Plant Cell 18:3635-3646.

Webster, R. K., and Gunnell, P. S. 1992. Rice blast. Pages 14-17 in: Compendium of Rice Diseases. R. K. Webster and P. S. Gunnell, eds. American Phytopathological Society, St. Paul, MN. 